\title{
Trypanosoma cruzi: Compared Vectorial Transmissibility of Three Major Clonal Genotypes by Triatoma infestans
}

\author{
Marta de Lana, ${ }^{1}$ Artur da Silveira Pinto, ${ }^{2}$ Christian Barnabé, Virginie Quesney, Sébastien Noël, \\ and Michel Tibayrenc \\ Centre d'Etudes sur le Polymorphisme des Microorganismes (CEPM), Unité Mixte de Recherche No. 9926 \\ Centre National de la Recherche Scientifique (CNRS)/Institut Français de Recherche Scientifique pour \\ le Developpement en Cooperation (ORSTOM), ORSTOM, BP 5045, 34032 Montpellier, Cedex 1, France
}

de Lana, M., da Silveira Pinto, A., Barnabe, C., Quesney, V., Noel, S., and Tibayrenc, M. 1998. Trypanosoma cruzi: Compared vectorial transmissibility of three major clonal genotypes by Triatoma infestans. Experimental Parasitology 90, 20-25. Twenty Trypanosoma cruzi stocks attributed to the 19/20,32, and 39 major clones (Tibayrenc et al. 1986) were used to infect experimentally third instar larvae of Triatoma infestans. Three variables were considered: (i) percentage of infected insects; (ii) number of flagellates per insect (NFI); and (iii) percentage of metacyclic trypomastigotes per insect. Differences between the genotypes under study for all parameters considered were detected. These differences were statistically significant $\left(P<10^{-3}\right)$, except between the 39 and 32 clonal genotypes for the NFI parameter. The correlation coefficient between the genetic distance and the biological parameters determined by the nonparametric Mantel's test was strongly significant $\left(P<10^{-4}\right)$. Data obtained suggest clearly that populations of parasites belonging to the 19/20 genotype are more efficiently transmitted (high transmissibility genotype) by the vector than the 32 genotype (low transmissibility genotype), while the 39 genotype presents intermediary characteristic. Results confirm the working hypothesis that the subdivision of $T$. cruzi into discrete clonal lineages has an impact on the vectorial competence of T. infestans, the most important vector of the chagasic infection in South America, and that different clonal lineages do not exhibit the same vectorial transmissibility. This fact is relevant both for Chagas' disease epidemiology and for the use of xenodiagnosis. (c) 1998 Academic Press

${ }^{1}$ Permanent address: Departamento de Análises Clínicas, Escola de Farmacia, Rua Costa Sena, Universidade Federal de Ouro Preto, Ouro Preto, MG, Brasil, CEP 35.400-000.

${ }_{1}^{2}$ Permanent, address: Departamento de Microbiologia, Instituto de Ciencias Biologicas, CP 486, Universidade Federal de Minas Gerais, 31270-010, Belo Horizonte, MG, Brasil.
Index Descriptors and Abbreviations: Trypanosoma cruzi; phylogenetic divergence; clonal structure; biological variability; multilocus enzyme electrophoresis (MLEE); random amplification of polymorphic DNA (RAPD); percentage of infected insects (\%II); number of flagellates per insect (NFI); percentage of metacyclic trypomastigotes per insect (\%DIF).

\section{INTRODUCTION}

Chagas' disease remains a major public health problem in Latin America. Its causative agent, the protozoan Trypanosoma cruzi, undergoes a complex life cycle passing through both triatomine bugs and mammals. Within the gut of the vector, the ingested bloodstream trypomastigotes undergo differentiation into proliferative epimastigotes that become infective metacyclic trypomastigotes in the rectum (Brener 1973). The relationships between the parasite's diversity and its vectorial transmissibility are not well known.

Studies have shown that $T$. cruzi populations differ in their ability to survive, multiply, and differentiate in the insect vector (Urdaneta-Morales and Rueda 1977; Garcia and Dvorack 1982; Schaub 1989; Mello et al. 1996).

Apart from vectorial transmissibility, the diversity of $T$. cruzi populations has been explored by general biological behavior (Andrade 1976), growth kinetic (Dvorak et al. 
1980), isoenzyme variability (Miles et al. 1980; Andrade et al. 1983; Tibayrenc and Ayala 1988), pathogenic properties (Miles et al. 1981), kinetoplast DNA restriction fragment polymorphism (Morel et al. 1980), and random amplified polymorphic DNA (RAPD) (Steindel et al. 1993; Tibayrenc et al. 1993).

Population genetic analyses have shown that $T$. cruzi has a typical clonal population structure (Tibayrenc et al. 1986; Tibayrenc and Ayala 1988). Among the natural clones evidenced by genetic markers, some are widespread and much more frequently sampled than others. They have been named: "major clones" (Tibayrenc and Ayala 1988; Tibayrenc and Breniere 1988). Moreover, it has been shown that clonal variability is statistically linked to relevant biological parameters such as culture growth, virulence in mice, and in vitro drug sensitivity (Laurent et al. 1997; Revollo et al. in press).

The present work aims at exploring the hypothesis that T. cruzi clonal diversity has an impact on its transmissibility through insect vectors. More specifically, as for previous experimental studies ((Laurent et al. 1997; Revollo et al. in press), the working hypothesis proposed here is that $T$. cruzi biological diversity (here: vectorial transmissibility) is statistically correlated to the phylogenetic divergence that is observed among the parasite's natural clones.

\section{MATERIALS AND METHODS}

(1) Parasites. The same standardized sample used for previous experimental studies (Laurent et al. 1997; Revollo et al. in press) has been used. All stocks have been fully characterized by both MLEE with 22 different genetic loci and RAPD (Tibayrenc et al. 1993). They have been cloned in the laboratory, with verification under the microscope. Information on the laboratory code, host, and geographic origin of the stocks under survey is given in Table I. This set of 20 stocks is representative of three major clonal lineages of $T$. cruzi, numbered 19/20, 32, and 39, according to the coding by Tibayrenc et al. (1986). Major clones 19, 20, 32, and 39 characterized by 15 enzyme loci (Tibayrenc et al. 1986) showed limited additional variability with the use of more discriminative markers (Tibayrenc et al. 1993), as expected. They should be considered as families of closely related clones rather than actual clones (Tibayrenc and Ayala 1988). Moreover, the $19 / 20$ group, formerly distinguished into 19 and 20 natural clones on the basis of 15 isoenzyme loci (Tibayrenc et al. 1986), is less clearly separated with more discriminative methods (Tibayrenc et al. 1993). In the present study, they are plotted together into a unique group of closely related clonal genotypes, designated 19/20. The three categories of clonal genotypes, 19/20, 32, and 39, illustrate different phylogenetic relationships: 32 and 39 are more closely related to each other, while 19/20 is more distantly related to both 32 and 39 (see Fig. 1).
(2) Experimental conditions. Experiments were undertaken with third instar nymphs of $T$. infestans reared in laboratory conditions, i.e., at about $26-27^{\circ} \mathrm{C}, 65-70 \%$ relative humidity, and allowed to feed on chickens every 3 weeks. The insects originate from an outbred colony with individuals coming from Chile, Uruguay, and Brazil. The insects were exposed to infection by using middle-log phase cultures forms from LIT medium, at $28^{\circ} \mathrm{C}$ in an artificial xenodiagnosis device through latex membranes. Eight milliliters of parasite suspension in citrated mice blood, at the final concentration of $5.0 \times 10^{5}$ cells $/ \mathrm{ml}$, was used. The system was maintained at $37^{\circ} \mathrm{C}$ and continuously homogenized with a magnetic stir bar. Only engorged insects were considered.

For parasite quantification, 30 days later, the whole digestive tube was removed and gently ground in 0.6-ml Eppendorf tubes containing $10 \mu 1$ of phosphate-buffered saline (PBS, pH 7.2). The suspension was then homogenized and used to prepare fresh slide smears covered with $22 \times 22$ lids. Preparations were scored microscopically for the presence of flagellates, total number of flagellates, and percentage of metacyclic trypomastigotes. Two hundred fields for each preparation were examined. Additionally, the entire intestinal tract of two insects for each stock was aseptically removed and introduced into $15-\mathrm{ml}$ Falcon tubes containing $3.0 \mathrm{ml}$ of LIT medium with $50 \mu \mathrm{g} / \mathrm{ml}$ of gentamicin. The tubes were kept at $28^{\circ} \mathrm{C}$ and weekly examined for the presence of flagellates. The clonal genotype of these isolates was verified by isoenzymes. Cellulose acetate electrophoresis was performed under conditions previously described (Ben Abderrazak et al. 1993). Five enzyme systems were assayed: phosphoglucose isomerase (EC 5.3.1.9, PGI); phosphoglucomutase (EC 5.4.2.2, PGM); isocitrate dehydrogenase (EC 1.1.1.42, IDH); 6-phosphogluconate dehydrogenase (EC 1.1.1.44, 6PGD); and glutamate oxalate transaminase (EC 2.6.1.1, GOT). This was sufficient to distinguish among the three groups of clonal genotypes surveyed here.

(3) Data analysis. For each stock, three variables were considered: (i) the percentage of infected insects (\%II); (ii) the number of flagellates per insect (NFI), and the percentage of metacyclic trypomastigotes per insect (\%DIF). These variables were estimated on the 20 stocks representative of the 19/20,32, and 39 clonal genotypes of T. cruzi (see Table I). At least 30 insects were exposed to each stock. A total of 620 engorged insects was considered. The reduced deviation test (SAS Program, version 5-06) was used to compare the parameters between the three categories of clonal genotypes (19/20, 32, and 39). Comparisons of percentages were used in the case of the \% II parameter, while comparisons of means were used in the case of the two other parameters.

Moreover, for each possible pair of stocks (190 pairwise comparisons), the absolute difference for the quantified result of each biological parameter was evaluated. An overall "biological distance" was calculated as follows. For each biological parameter, the highest value of absolute difference in all pairs of stocks was 1 . The lowest value was 0 . The other values were expressed in percentages of the highest value. For each pair of stocks, the overall "biological distance" was given by the arithmetic means of all the values obtained according to this procedure for the three biological parameters under study. This procedure gives an equal weight to each biological parameter in the overall biological distance. Correlations between this overall biological distance, on one hand, and genetic distances measured from either MLEE or RAPD analysis, on the other hand, were then evaluated with a nonparametric Mantel test (Mantel 1967). Briefly, this test is based on a Monte Carlo simulation with $10^{4}$ iterations, which randomly permutes 
TABLE I

List of the 20 Trypanosoma cruzi Stocks Under Study

\begin{tabular}{|c|c|c|c|}
\hline Stock & Country & Place & Host \\
\hline SP104cl1 (19/20) & Chile & IV Region & Triatoma spinolai \\
\hline Cutia cl4 (19/20) & Brazil & Espirito Santo & Dasylprocta agudi \\
\hline Gamba cl1 (19/20) & Brazil & Sao Paulo & Didelphis azarae \\
\hline 13379 cl7 (19/20) & Bolivia & Santa Cruz & Man, acute form \\
\hline OPS21 cl11 (19/20) & Venezuela & Cojedes & Man \\
\hline SO34 cl4 (19/20) & Bolivia & Toropalka (Potosi) & Triatoma infestans \\
\hline Cuica cl1 (19/20) & Brazil & Sao Paulo & Opossum cuica philander \\
\hline $\mathrm{P} / 209$ cl1 $(19 / 20)$ & Bolivia & Sucre & Man, chronic form \\
\hline Esquilo cl1 (19/20) & Brazil & Sao Paulo & Sciurus aestuans ingramini \\
\hline $\mathrm{P} / 11$ cl2 $(19 / 20)$ & Bolivia & Cochabamba & Man, chronic form \\
\hline SC43 cl1 (39) & Bolivia & Santa Cruz & Triatoma infestans \\
\hline Bug2148 cl1 (39) & Brazil & Rio Grande do Sul & Triatoma infestans \\
\hline Bug2149 cl1 (39) & Brazil & Rio Grande do Sul & Triatoma infestans \\
\hline $\mathrm{SO} 3 \mathrm{cl} 5(39)$ & Bolivia & Otavi (potosi) & Triatoma infestans \\
\hline MN cl2 (39) & Chile & IV Region & Man, chronic form \\
\hline MAS1 cl1 (32) & Brazil & Brasilia & Man \\
\hline CBB cl3 (32) & Chile & Tulahen & Man \\
\hline Tu18 cl2 (32) & Bolivia & Tupiza & Triatoma infestans \\
\hline IVV cl4 (32) & Chile & Santiago & Man, chronic form \\
\hline MVB cl8 (32) & Chile & Santiago & Man, chronic form \\
\hline
\end{tabular}

Note. Clonet number, (in parentheses) refers to the major clonal genotypes previously identified by multilocus enzyme electrophoresis with 15 genetic loci (Tibayrenc and Ayala 1988).

the different cells of one of the distance matrices. Different from the classical correlation test, this randomization procedure does not need any assumptions about the number of degrees of freedom.

\section{RESULTS}

In the experimental conditions used, it was observed intuitively that the three different clonal genotypes of $T$. cruzi surveyed here tend to behave differently in T. infestans, i.e., stocks belonging to distinct genotypes of the parasite differ in their ability to complete the life cycle in the digestive tract of the insect vector. This was fully confirmed by statistical analysis, although the standard deviations of the values recorded were high (see Table II). All comparison tests between the three different clonal genotypes based on the reduced deviation statistics were highly significant $(P<$ $10^{-3}$ ), except in the case of the comparison between the stocks attributed to the 32 and 39 genotypes for the NFI parameter, which showed no significant differences.

The general tendency was that the group 19/20 showed the highest values, the group 32 showed the weakest values, and the 39 group showed intermediary values.
The correlation coefficient between the genetic distances and the biological differences estimated by the Mantel test was also strongly significant $\left(<10^{-4}\right)$. This shows that those stocks that are genetically related to each other tend to behave similarly in $T$. infestans, which is contrary to those stocks that are genetically distantly related.

Lastly, the isoenzyme profiles of all the stocks under survey showed no change after passage through the insect vector (data not shown).

\section{DISCUSSION}

The set of stocks under survey represents a convenient sample for testing the hypothesis of linkage between phylogenetic diversity of $T$. cruzi natural clones and a relevant biological property of the parasite, namely its transmissibility through a major vector species, $T$. infestans. The results show the same overall pattern from previous studies of our group dealing with the same sample of stocks and other biological parameters (Laurent et al. 1997; Revollo et al. in press). First, within each genotype category taken separately, 


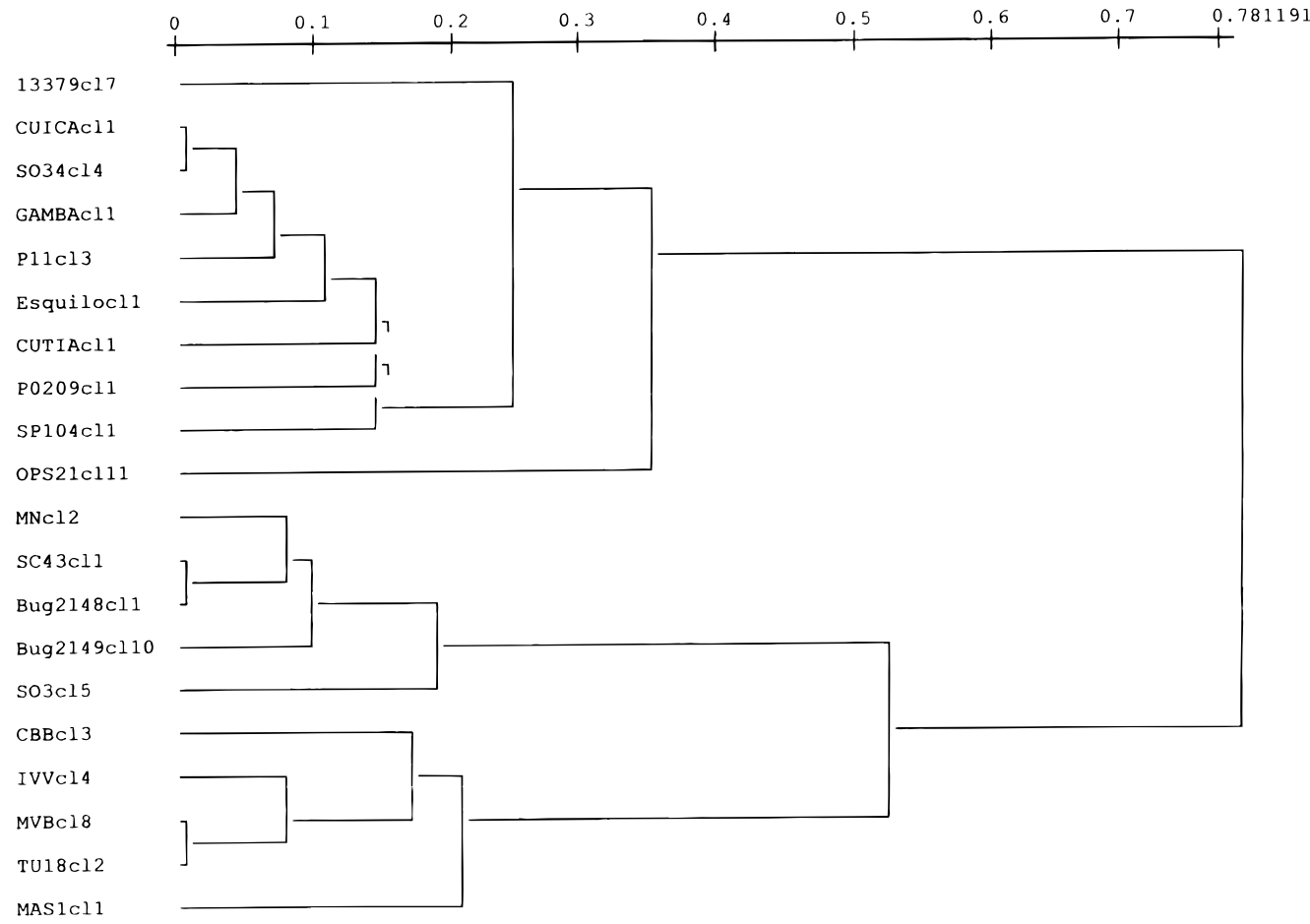

FIG. 1. An UPGMA (unweighted pair-group method with arithmetic averages) dendrogramme (Sneath and Sokal 1973) depicting the phylogenetic relationships among the 20 Trypanosoma cruzi stocks under study, assayed by 22 isoenzyme loci (Tibayrenc et al. 1993). Top cluster corresponds to the clonal genotype 19/20, medium cluster corresponds to the clonal genotype 39, and bottom cluster corresponds to the clonal genotype 32 (genotype numbering after Tibayrenc and Ayala 1988). The scale indicates genetic distances estimated with the index of Jaccard (1908).

there is a notable variability of the biological parameters, as evidenced by the strong values of standard deviation for the NFI and \%DIF parameters (see Table II). This means that the stocks pertaining to a given clonal genotype are not homogeneous for these biological parameters. An additional, possible explanation for this result is that the insect vectors used are themselves genetically heterogeneous, which could interfere with their vectorial competence. Second, despite these notable standard deviations, there is a strong statistical correlation between phylogenetic divergence among $T$. cruzi natural clones on one hand and biological differences (here: transmissibility through $T$. infestans) on the other hand. This is confirmed by both (i) pairwise comparison by the reduced deviation test of clonal genotypes for each parameter taken separately, and (ii) overall correlation evaluated by the Mantel test between genetic distances (phylogenetic divergences) on one hand and overall biological differences on the other hand. Especially remarkable is the different ability of the three clonal genotypes to differentiate into infective metacyclic trypomastigotes, a property that conditions potentially their ability to infect mammalian hosts including humans.
TABLE II

Average Values of the Three Biological Parameters Measured for the Transmissibility of the 20 Trypanosoma cruzi Stocks (see Table I) through Triatoma infestans

\begin{tabular}{lccc}
\hline Clonal genotype & $\%$ II & NFI & $\%$ DIF \\
\hline $19 / 20$ & 81.76 & $103,716 \pm 201,048$ & $10.48 \pm 16.60$ \\
39 & 68.12 & $51,144 \pm 138,132$ & $5.84 \pm 11.95$ \\
32 & 51.33 & $31,380 \pm 91,560$ & $0.51 \pm 2.27$ \\
\hline
\end{tabular}

Note. \%II, percentage of infected insects; NFI number of flagellates per insect; \%DIF percentage of metacyclic trypomastigotes per insect.

This value was high for the 19/20 clonal genotype, and very weak for the 32 genotype, while the 39 genotype showed intermediary values (see Table II). More generally, this study confirms a notable tendency of previous ones (Laurent et al. 1997; Revollo et al. in press). By comparison with stocks attributed to clonal genotypes 32 and 39, stocks attributed to clonal genotype 19/20 (i) grow more quickly in in vitro culture; (ii) differentiate more easily in culture cells; 
(iii) infect more easily mice, and are more virulent for them; (iv) are less sensitive in vitro to Rochagan and Nifurtimox; (v) show higher transmissibiliy through $T$. infestans (the present study).

In the case of the present study, it is obvious that different transmissibility and different ability to differentiate into infective trypomastigote forms have a potential impact on both transmission cycles and the routine use of xenodiagnosis. Since the repartition of $T$. cruzi clonal genotypes differs among endemic countries (Tibayrenc and Ayala 1988), the transmission patterns could be modified by the clonal genotypes present in given areas, as well as the sensitivity of the xenodiagnosis.

Our work confirms that it is misleading to consider T. cruzi as a homogeneous entity and that phylogenetic divergence among T. cruzi natural clones is a parameter that deserves to be taken into account for all applied studies dealing with this parasite.

\section{ACKNOWLEDGMENTS}

This work was supported by the WHO Special Programme for Research and Training in Tropical Diseases (No. 910268), an EEC STD3 Grant No. TS3* CT92-0155, and a Groupement de Recherche Grant CNRS/French Army. M. De Lana and A. Pinto were supported by a fellowship from CNPq, Brazil.

\section{REFERENCES}

Andrade, S. 1976. Tentative for grouping different Trypanosoma cruzi strains in some types. Revista del Instituto de Medicina Tropical de Sao Paulo 18, 140-141.

Andrade, V., Brodskin, C., and Andrade, S. G. 1983. Correlation between isoenzyme patterns and biological behaviour of different strains of Trypanosoma cruzi. Transactions of the Royal Society of Tropical Medicine and Hygiene 77, 796-799.

Ben Abderrazak, S., Guerrini, F., Mathieu-Daudé, F., Truc, P., Neubauer, K., Lewicka, K., Barnabe, C., and Tibayrenc, M. 1993. Isozyme electrophoresis for parasite characterization. In "Protocols in Molecular Parasitology" (J. E. Hyde, Ed.), Vol. 21, NJ, Humana Press, Totowa, pp. 361-382.

Brener, Z. 1973. Biology of Trypanosoma cruzi. Annual Review of Microbiology 27, 347-382.

Dvorak, J. A., Hartman, D. L., and Miles, M. A. 1980. Trypanosoma cruzi: Correlation of growth kinetics to zymodeme type in clones derived from various sources. The Journal of Protozoology 27, 472474.
Garcia, E. S., and Dvorak, J. A. 1982. Growth and development of two Trypanosoma cruzi clones in the arthropod Dipetalogaster maximus. The American Journal of Tropical Medicine and Hygiene 31, 259-262.

Jaccard, P. 1908. Nouvelles recherches sur la distribution florale. Bulletin de la Societe vaudoise de Sciences Naturelles 44, 223-270.

Laurent, J. P., Barnabe, C., Quesney, V., Noel, S., and Tibayrenc, M. 1997. Impact of clonal evolution on the biological diversity of Trypanosoma cruzi. Parasitology 114, 213-218.

Mantel, N. 1967. The detection of disease clustering and a generalised regression approach. Cancer Research 27, 209-220.

Mello, C. B., Azambuza, P., Garcia, E. S., and Ratcliffe, N. A. 1996. Differential in vitro and in vivo behaviour of three strains of Trypanosoma cruzi in the gut and hemolymph of Rhodnius prolixus. Experimental Parasitology 82, 112-121.

Miles, M. A., Lanham, S. M., De Sousa, A. A., and Povoa, M. 1980. Further enzymatic characters of Trypanosoma cruzi and their evaluation for strain identification. Transactions of the Royal Society of Tropical Medicine and Hygiene 74, 221-237.

Miles, M. A., Cedillos, R. A., Povoa, M. M., Prata, A., Sousa, A. A., and Macedo, V. 1981. Do radically dissimilar Trypanosoma cruzi strains (zymodemes) cause Venezuelan and Brazilian forms of Chagas's disease? The Lancet 20, 1338-1340.

Morel, C., Chiari, E., Camargo, E. P., Mattei, D. D., Romanha, A. J., and Simpson, L. 1980. Strains and clones of Trypanosoma cruzi can be characterized by patterns of restriction endonuclease products of kinetoplast DNA minicircles. Proceedings of the National Academy of Science USA 77, 6810-6814.

Revollo, S. Oury, B., Laurent, J. P., Barnabe, C., Quesney, V., Carriere, V., Noel, S., and Tibayrenc, M. Trypanosoma cruzi: Impact of clonal evolution of the parasite on its biological and medical properties. Experimental Parasitology, in press.

Schaub, G. A. 1989. Trypanosoma cruzi: Quantitative studies of development of two strains in small intestine and rectum of the vector Triatoma infestans. Experimental Parasitology 68, 260-273.

Sneath, P. H. A., and Sokal, R. R. 1973. In Numerical Taxonomy. The principle and practice of numerical classification (D. Kennedy and R. B. Park, Eds.), pp 537. Freeman, San Francisco.

Steindel, M., Dias Neto, E., de Menezes, C. L. P., Romanha, A. J., and Simpson, A. J. G. 1993. Random amplified polymorphic DNA analysis of Trypanosoma cruzi strains. Molecular and Biochemical Parasitology 60, 71-80.

Tibayrenc, M., Ward, P., Moya., A, and Ayala, F. J. 1986. Natural populations of Trypanosoma cruzi, the agent of Chagas' disease, have a complex multiclonal structure. Proceedings of the National Academy of Science USA 83, 115-119.

Tibayrenc, M., and Ayala, F. J. 1988. Isoenzyme variability in Trypanosoma cruzi, the agent of Chagas' disease: Genetical, taxonomical, and epidemiological significance. Evolution 42, 277-292.

Tibayrenc, M., and Breniere, S. F. 1988. Trypanosoma cruzi: Major clones rather than principal zymodemes. Memorias do Instituto Oswaldo Cruz Rio de Janeiro 83, 249-255.

Tibayrenc, M., Neubauer, K., Barnabé, C., Guerrini, F., Scarecky, D., and Ayala, F. J. 1993. Genetic characterization of six parasitic proto- 
zoa: Parity between random-primer DNA typing and multilocus enzyme electrophoresis. Proceedings of the National Academy of Science USA 90, 1335-1339.

Urdaneta-Morales, S., and Rueda, S. 1977. A comparative study of the behaviour of Venezuelan and Brazilian strians of Trypanosoma
(Schizotrypanum) cruzi in the Venezuelan invertebrate host $\_$Rhodnius prolixus). Revista do Instituto de Medicina Tropical Sao Paulo 19, 241-250.

Received 1 October 1997; accepted with revisions 30 March 1998 